\title{
EFEITOS DOS SURFACTANTES ANIÔNICOS LAS E LESS NO DESENVOLVIMENTO E NA MORFOLOGIA DA CIANOBACTÉRIA PLANKTOTHRIX AGARDHII CCIBt3125
}

\author{
Santos, V.M. ${ }^{1 *}$; Santos, K.R.S. ${ }^{2}$; Carvalho, L.R. ${ }^{1}$ \& Sant'Anna, C.L. ${ }^{1}$ \\ 1- Instituto de Botânica (São Paulo), Secretaria do Meio Ambiente, Núcleo de Pesquisa em Ficologia. \\ 2- Instituto Federal de Educação, Ciência e Tecnologia de Santa Catarina (IFSC). \\ *Corresponding author: valdilenemaria@yahoo.com.br
}

\begin{abstract}
Santos, V.M.; Santos, K.R.S.; Carvalho, L.R.; Sant'Anna, C.L. 2014. Efeitos dos Surfactantes Aniônicos Las e Less no Desenvolvimento e na Morfologia da Cianobactéria Planktothrix Agardhii CCIBt3125. Braz. J. Aquat. Sci. Technol. 19(1): 1-5. eISSN 1983-9057. DOI: 10.14210/bjast.v19n1.p1-5 The anionic surfactants Linear Alkylbenzene Sulfonate (LAS) and Sodium Lauryl Ether Sulfate (SLES) are the main compounds used in commercial detergent formulations. These compounds are released into the sewer and then may pollute rivers and springs, which are inhabited by thousands of microorganism, among which are cyanobacteria. Cyanobacteria are prokaryotes capable of oxygenic photosynthesis and of synthesizing numerous compounds of environmental and pharmacological relevance. The aim of this study was to evaluate the effects of surfactants LAS and SLES on the growth and morphology of the cyanobacterial strain Planktothrix agardhii CCIBt3125. The experiment was carried out for 16 days and trichomes were counted every two days in a Fuchs Rosenthal chamber. The BG-11 medium was used as control and the following treatments were tested $(n=3)$ : BG- $11+4 \%$ LAS (concentration $4,13 \mathrm{mM}$ ) and BG-11 $+4 \%$ SLES (concentration $5,32 \mathrm{mM}$ ), kept in a temperature of $23 \pm 1^{\circ} \mathrm{C}$, photoperiod 14/10 light/dark cycle and light intensity 40-50 $\mu \mathrm{mol}$ photons $\mathrm{m}-2 . \mathrm{s}-1$. Under controlled conditions, the culture growth rate was 0.17 ( $\mu$.dia-1), and the exponential phase occurred between 2 to 10 days, whereas the addition of LAS and SLES promoted inhibitory effects: on the tenth day, a reduction of $69 \%$ in the trichome density was observed for treatment with LAS, and one of $70 \%$, for SLES, and on the sixteenth day, a reduction of $88 \%$ (LAS) and $90 \%$ (SLES), respectively. To our knowledge, our results are the first reporting on these surfactants inhibitory effects over P. agardhii and are in accordance with literature data about other cyanobacterial species; furthermore, they show the need to better control the wastewater disposal in order to avoid negative effects on aquatic biota.
\end{abstract}

Key words: Cyanobacteria, development in culture, detergents.

\section{INTRODUÇÃO}

Os surfactantes - também conhecidos como tensoativos - são constituídos por moléculas anfifílicas (ou anfipáticas), cujas estruturas apresentam duas regiões distintas, uma polar (hidrofílica) e outra não polar (hidrofóbica - lipofílica); esta propriedade permite a solubilização ou emulsificação de materiais insolúveis e a subsequente formação de um filme ordenado na interface entre dois fluídos imiscíveis tais como ar-água, óleo-água, ou mesmo, entre sólido-água (Borsato et al., 2009). A fase hidrofílica associa-se à água, por pontes de hidrogênio e a fase lipofílica, às gorduras, por interações de van der Waals (Souza, 2006).

Os surfactantes podem ser iônicos ou não iônicos. Os iônicos são classificados em aniônicos (carga negativa), catiônicos (carga positiva) ou anfóteros (duas cargas iônicas opostas). A carga iônica permitirá ao surfactante interagir com outras moléculas e com microorganismos (Louvado, 2010).

Algumas dessas interações, principalmente com organismos aquáticos, estão documentadas em estudos que avaliaram o efeito inibitório dos surfactantes sobre o desenvolvimento de bactérias e de cianobac- térias (Zagatto et al., 1987; Yan, 1998; Barbieri, 2008; Lima \& Etchegaray 2011; Carraschi et al., 2012); seu efeito deletério sobre paredes celulares microbianas (Glover, 1999; Louvado, 2010); e a sua capacidade de remover cianotoxinas das águas (Lima \& Etchegaray, 2011). As publicações existentes sobre o assunto não exaurem a diversidade dos possíveis efeitos dos tensoativos sobre os incontáveis microorganismos que habitam determinado ambiente.

Nosso interesse nas ações dos surfactantes aniônicos sobre microorganismos procariontes levou-nos à observação da influência do Linear Alquilbenzeno Sulfonato (LAS) e do Lauril Éter Sulfato de Sódio (LESS) sobre a cianobactéria Planktothrix agardhii (Gomont) Anagnostidis \& Komákek. Estes dois tensoativos são amplamente utilizados nas indústrias farmacêutica, de artigos para higiene pessoal e de domisanitários, e os resíduos por eles gerados são habitualmente descartados nas redes de esgoto, contaminando rios e mananciais (Barbieri, 2008).

A espécie filamentosa planctônica P. agardhii é encontrada principalmente em corpos d'água e em reservatórios de abastecimento público de praticamente todo o território brasileiro (Sant'Anna et al., 2003; Silva, 
2009, Santos \& Sant'Anna, 2010). É capaz de formar florações e apresenta aerótopos (vesículas de gás associadas à flutuação), além de produzir uma expressiva quantidade de substâncias biologicamente ativas, como algumas variantes de microcistinas (Sant'Anna et al., 2003; Tonk et al., 2005; Marie et al., 2012), anabaenopeptinas, aeruginosinas e microviridinas, todas elas potentes inibidores de serino-proteases, que são enzimas envolvidas em processos fisiológicos críticos, tais como digestão protéica e regulação da pressão arterial e da coagulação sanguínea (Hedstrom, 2002).

O objetivo deste trabalho foi avaliar a influência dos surfactantes aniônicos LAS e LESS no desenvolvimento e na morfologia da cianobactéria Planktothrix agardhii CCIBt3125 mantida em cultivo e discutir as prováveis consequências destes efeitos.

\section{MATERIAIS E MÉTODOS}

A cepa Planktothrix agardhii CCIBt3125 foi coletada no Lago das Garças, corpo d'água eutrófico situado no Jardim Botânico de São Paulo, Parque Estadual das Fontes do Ipiranga (PEFI), SP, no dia primeiro de março de 1999. Esta cepa é mantida na Coleção de Culturas do Instituto de Botânica, nas seguintes condições: temperatura $23^{\circ} \mathrm{C} \pm 1$; fotoperíodo 14/10 luz/escuro; irradiância 40-50 $\mu \mathrm{mol}$ fótons m2. s-1 (Azevedo \& Sant'Anna, 2003).

Para a realização do presente estudo, um inóculo $(10 \mathrm{~mL})$ de $P$. agardhii CClBt3125 foi retirado diretamente do Banco de Culturas e transferido para frasco erlenmeyer de $250 \mathrm{~mL}$, contendo $100 \mathrm{~mL}$ de meio BG-11 líquido. Este material permaneceu em cultivo durante sete dias, nas mesmas condições do Banco de Cultura, sendo então, utilizado como inóculo para os diferentes tratamentos em triplicata que compunham o experimento: Controle: meio BG-11 (100 $\mathrm{mL}$ ); LAS: BG-11 + LAS (4\%): $96 \mathrm{~mL}$ de meio BG-11 + $4 \mathrm{~mL}$ de LAS (concentração 4,13 mM); e LESS: BG-11 + LESS (4\%): $96 \mathrm{~mL}$ de meio BG-11 + $4 \mathrm{~mL}$ de LESS (concentração 5,32 mM).

Foram coletadas alíquotas de $700 \mu \mathrm{L}$ de cada triplicata dos tratamentos e do controle, a cada dois dias, aproximadamente às 16 horas, em capela de fluxo laminar, durante 16 dias. Estas amostras foram fixadas com $700 \mu \mathrm{L}$ de formol a $8 \%$ e armazenadas em microtubos de $1,5 \mathrm{~mL}$, até a análise. A contagem dos tricomas foi feita em câmara de Fuchs Rosenthal em microscópio óptico binocular Zeiss Primo Star, com ocular micrometrada, no aumento de 100x. Os dados coletados estão apresentados em densidade de tricomas. $\mathrm{mL}-1$. Para análise estatística, os dados de todos os tratamentos foram submetidos à análise de variância (ANOVA) de um fator e teste Tukey (Programa GraphPad Prism versão 5.0), sendo consid- erados significativos se $p<0,05$ (Santos et al., 2011). Fotografias foram obtidas com auxílio de câmera digital acoplada à ocular do microscópio.

A condutividade elétrica e o potencial hidrogeniônico dos materiais contidos nos frascos Controle, LAS e LESS foram avaliados, utilizando-se condutívimetro Digimed Dm-32 e pHametro Digimed Dm-20, respectivamente; os meios utilizados no experimento foram previamente esterilizados, em autoclave, por 30 minutos, a $120^{\circ} \mathrm{C}$ e $1,2 \mathrm{~atm}$. As transferências de biomassa foram realizadas em capela de fluxo laminar, em ambiente axênico.

\section{RESULTADOS E DISCUSSÃO}

As concentrações das soluções resultantes da adição de LAS e de LESS ao meio de cultura são superiores aos valores das concentrações micelares críticas de ambos os surfactantes que são de 0,82 mM para o LESS e 1,64 mM para o LAS, não estando, portanto, ambos igualmente na forma monomérica (Cirin et al., 2011; Al-Soufi et al., 2012). No entanto, as concentrações selecionadas são menores que as encontradas no meio ambiente (Scott et al. 2000; Penteado et al., 2006; Frinhani \& Carvalho, 2010), e nossos resultados evidenciam que mesmo em baixas concentrações os surfactantes testados foram nocivos à cianobactéria Planktothrix agardhii CCIBt3125 (Figura 1).

Na figura 1, a comparação entre as curvas de crescimento da cepa, nas diferentes condições, mostra que a adição de LAS e de LESS ao meio de cultivo teve efeito inibitório sobre o desenvolvimento de Planktothrix agardhii, pois no tratamento controle, a cepa apresentou crescimento exponencial do segundo ao décimo dia, com taxa máxima de 0,17 ( $\mu$ dia- 1 ), enquanto que nos tratamentos com os surfactantes não foi observada taxa de crescimento. Ainda em comparação com o controle, no décimo dia, o LAS e o LESS ocasionaram redução na densidade de tricomas de $69 \%$ e $70 \%$, e no décimo sexto dia, de $88 \%$ e $90 \%$, respectivamente (Figura 1), não havendo diferença significativa entre os surfactantes.

Os valores de $\mathrm{pH}$ após a adição de LESS e de LAS ao Meio BG-11 (Tabela 1) estão próximos ao valor do meio BG-11, ou seja, são levemente alcalinos e, portanto adequados ao desenvolvimento de $P$. agardhii (Hossain et al., 2012; Bittencourt-Oliveira et al., 2012). Por outro lado, os valores de condutividade elétrica após a adição de LESS e de LAS ao meio BG-11 foram cerca de duas vezes maiores que o controle (Tabela 1). De acordo com Santos \& Sant'Anna (2010), P. agardhii foi encontrada apenas em lagoas com amplitude de condutividade de 716 a $2.110 \mu$ S.cm-1, o que indica 


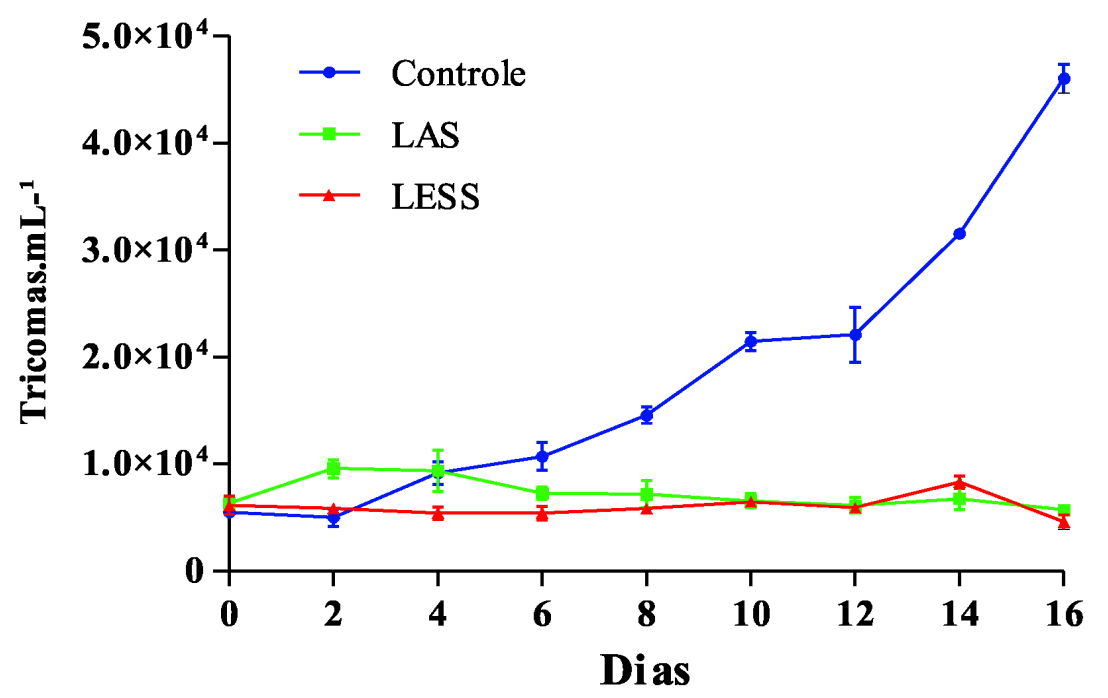

Figura 1. Curvas de crescimento de Planktothrix agardhii CCIBt3125 (tricomas. mL-1) obtidas na condição controle (meio BG-11) e para os tratamentos com os tensoativos aniônicos LAS e LESS.

que a condutividade elevada pode ser um fator limitante ao crescimento desta espécie.

A inibição do crescimento de cianobactérias causada por surfactantes já havia sido observada, dentre outras cianobactérias e algas (Lewis, 1990) - em Gloecapsa (Tözüm-Calgan \& Atay-Güneyman, 1994), em Synechococcus elongatus (Lima, 2010), e em Microcystis aeruginosa (Lima \& Etchegaray, 2011), porém não em Planktothrix, e foi considerada resultado da interação destas substâncias com proteínas, lipídeos e membranas destes organismos, inibindo ou alterando suas funções (Lewis, 1990; Yan et al. 1998). A desnaturação de proteínas da parede celular poderia ocasionar a alteração da permeabilidade aos nutrientes ou a lise celular, levando à diminuição dos tricomas (Lewis, 1990). Outra ação atribuída aos surfactantes é a capacidade de dissociação do complexo pigmento-proteina, observada em algas e em plantas, o que diminui a fotossíntese, e, consequentemente, o desenvolvimento dos organismos (Reunova \& Ayzdaycher, 2003; Branislav et al., 2010).

Entretanto, em nosso experimento, além desse, outros efeitos adversos foram observados: um deles foi a descoloração dos pigmentos (Figura 2) provavelmente resultante da dissociação do complexo pigmento-proteína (Branislav et al., 2010; Reunova \& Ayzdaycher, 2003). E o outro foi a perda dos aerótopos (Figura 2) importante alteração morfológica ainda não relatada para cianobactérias. Com a perda dos aerótopos, a cianobactéria torna-se incapaz de regular a flutuação na coluna d'água, e consequentemente sedimenta, o que aumenta o efeito negativo dos surfactantes testados sobre o desenvolvimento deste organismo.

Nossos resultados condizem com os observados para as outras espécies de cianobactérias (Tözüm-Calgan \& Atay-Güneyman, 1994; Lima, 2010 e 2011) e acrescentam novos dados ao conjunto de informações existentes sobre os efeitos dos surfactantes sobre esses organismos. Desta maneira há necessidade de maior controle na destinação de efluentes contendo detergentes devido aos seus efeitos negativos sobre a biota aquática e consequentemente sobre a qualidade ecológica das águas.

Tabela 1: Condutividade elétrica e potencial hidrogeniônico dos tratamentos.

\begin{tabular}{ccrc}
\hline \hline & Meio BG-11 & BG-11+ & BG-11+ \\
& Controle & LAS & LESS \\
\hline $\begin{array}{c}\text { Condutividade }\left(\mu \mathrm{s} . \mathrm{cm}^{-1}\right) \\
\mathrm{pH}\end{array}$ & 2.270 & 3.730 & 4.210 \\
& 7,5 & 7,3 & 7,6 \\
\hline \hline
\end{tabular}




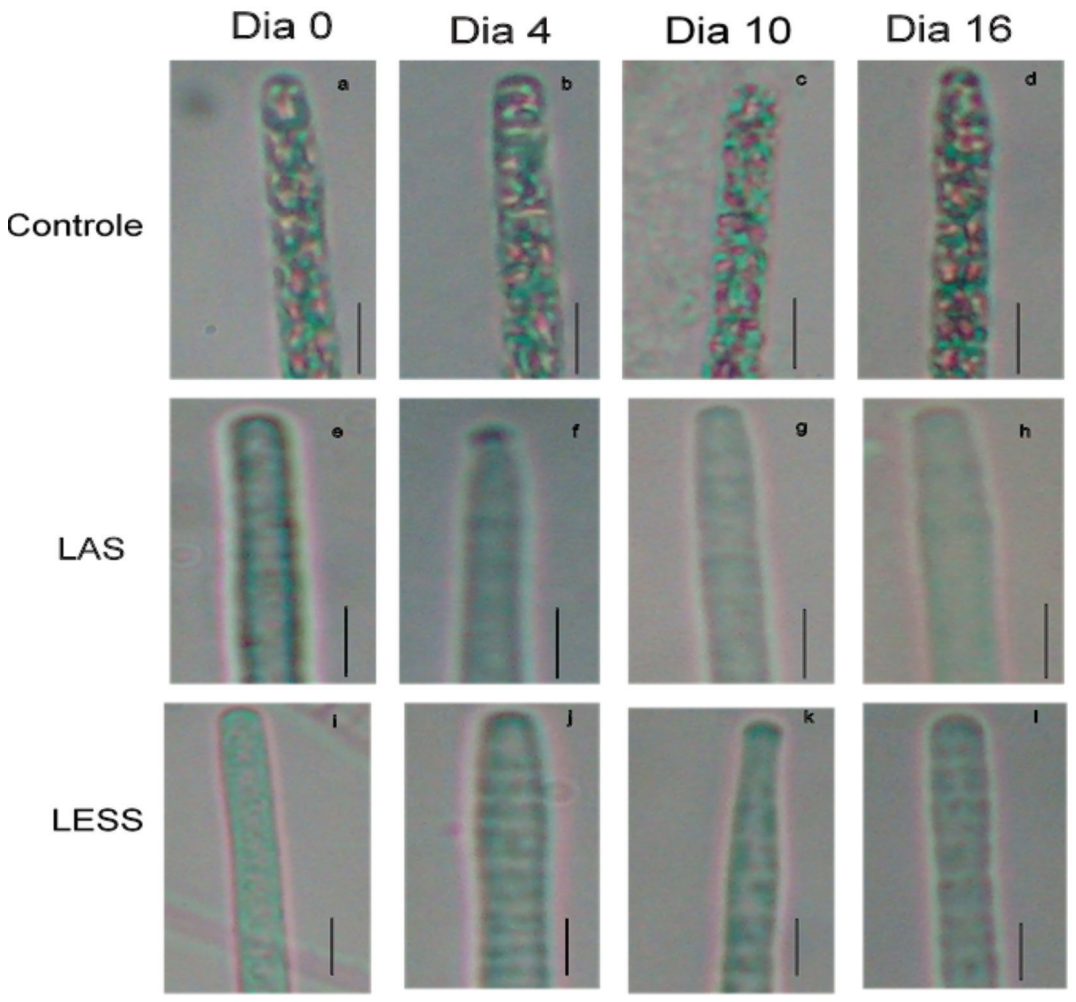

Figura 2: Aspecto dos tricomas de Plaktothrix agardhii CCIBt3125 na condição controle BG-11 (a-d) e nos tratamentos com os tensoativos aniônicos LAS (e-h) e LESS (i-I) durante 16 dias de cultivo. Escalas $=10 \mu \mathrm{m}$.

\section{REFERÊNCIAS}

Al-Soufi, W; Pineiro, L. \& Novo, M. 2012. A mode for monomer and micellar concentrations in surfactant solutions.Application to conductivity, NMR, diffusion and surface tension data. J.of Col.and Interf. Sci. 370(1): 102-110.

Azevedo, M.T.P. \& Sant'Anna, C.L. 2003. Sphaerocavum brasiliense, a new planktic genus and species of Cyanobacteria from reservoirs of São Paulo State, Braz. Algol. Studies. 109: 79-92.

Barbieri, E. 2008. Efeito dos Surfactantes DSS e LAS-C12 sobre o Camarão-rosa (Farfantepenaeus paulensi, Pérez-Farfante). Instituto de Pesca da Secretaria da Agricultura e Abastecimento do Estado de São Paulo Cananéia São Paulo, J. Braz. Soc. Ecotoxicol. 3 (1):35-40.

Bittencourt-Oliveira, M.C.; Dias, S.N. Moura, A.N.; Cordeiro-Araújo, M.K. \& Dantas, E.W.2012. Seasonal dynamics of cyanobacteria in a eutrophic reservoir (Arcoverde) in a semi-arid region of Brazil. Braz. J. of Biology. 72(3): 533-544.

Borsato, D. Moreira, I. \& Galão, O.F.2009. Detergentes Naturais e Sintéticos, um Guia Técnico. $2^{\mathrm{a}}$ edição revisada. Londrina: Paraná, p.175.

Branislav, R. Jovanić, B.R.; Bojović, S.; Panić, B. Radenković, B. \& Despotović, M. 2010. The effect of detergent as polluting agent on the photosynthetic activity and chlorophyll content in bean leaves.J. Health .2(5): 395-399.

Carraschi, S.P.; Luna,L.A.V; Neto, A.N.;Gírio,A.C.F.; Cruz,C.\&Pitelli R.A. 2012. Toxicidade aguda e risco ambiental de surfactantes agrícolas para o guaru Phalloceros caudimaculatus (Pices: Poecilidae). Centro de Aquicultura da Unesp/Caunesp: Campus de Jaboticabal, Brasil, São Paulo. J.Braz. Soc.Ecotoxicol. 7(1): 28-32.

Cirin, D.M. Posa, M.M. \& Krstonosic, V.S. 2011. Interactions between selected bile salts and Triton X-100 or sodium lauryl ether sulfate. J. Chem. Central.5: 01-08.

Frinhani, E.M. \& Carvalho, E.F. (2010). Monitoramento das águas do rio do tigre, joaçaba, SC. Uno. \& Ci. 1(1):49-58.

Glover,R.E.;Smith,R.R.;Jones,M.V.;Jackson,S.K. \& Rowlands, C.1999. An EPR investigation of surfactant action on bacterial membrances. FEMS Microbiol.Letters.177(1):57- 62. 
Hedstrom, L. 2002. Serine Protease Mechanism and Specificity.Chem. Rev.102 (12): 4501-4523.

Hossain, Y.;Jewel, A.S.;Fulanda, B.; Ahamed, F.;Rahman, S.;Jasmine, S. \& Ohtomi, J.2012. Dynamics of Cyanobacteria Planktothrix species (Oscillatoriales: Phormidiaceae) in Earthen Fish Ponds, Northwestern Bangladesh. Sains Malay. 41(3): 277-284.

Keil,C.; Forcherta,A.; Fastner, J.; Szewzyk, U.; Rotarrd,W.;Chorus,I.\& Kratke,R.2002. Toxicity and microcystin content of extracts from a Planktothrix bloom and two laboratory strains, Water Research Berlin Germany. 36 (8): 2133-2139.

Komárek, J. \& Anagnostidis, K. 2005. Cyanoprokariota, 2. Teil: Oscillatoriales. In: B. Büdel, G. Gärdner, L. Krienitz \& M. Schagul (eds.). Subwasserflora von mitteleuropa, Band 19/2. Spektrum Akademischur Verlag. 759p.

Lewis M.A. 1990. Chronic toxicities of surfactants and detergent builders to algae: a review and risk assessment. Ecotoxicol. Environ. Safety. 20(2): 123-140.

Lima, D.T.B. \& Etchegaray, A. 2011. Inibição do crescimento de Microcystis aeruginosa e extração de microcistina utilizando surfactantes. In Anais do XVI Encontro de Iniciação Científica da PUC-Campinas e I Encontro de Iniciação em desenvolvimento Tecnológico e Inovação da PUCcampinas. $6 \mathrm{p}$.

Louvado, O.D.A.M. 2010. Isolamento e Caracterização de Bactérias Resistentes a Surfactantes. Dissertação de Mestrado. Universidade de Aveiro. $75 p$.

Marie, B.; Huet, H.; Marie, A.; Djediat, C.; Puiseux-Dao, S.; Catherine, A.; Trinchet, I. \& Edery, M. 2012. Effects of a toxic cyanobacterial bloom (Planktothrix agardhii) on fish: Insights from histopathological and quantitative proteomic assessments following the oral exposure of medaka fish (Oryzias latipes). Aquatic Toxicol. 114-115:39-48.

Penteado, J.C.P.; Seound, O.A.E. \& Carvalho, L.R.F. 2006. Alquilbenzeno sulfonato linear: uma visão ambiental e analítica. Quim. nova. 26(5): 1038-1046.

Reunova, Y.A. \& Ayzdaycher, N.A. 2003. Effects of detergent on chlorophyll a content and quantity dynamics of microalga Chroomonas salina (Wils.) Butch. (Cryptophyta). Internat. J. Algae. 5(4): 90-97.

Santos, K.R.S.; Jacinavicius, F.R. \& Sant'Anna, C.L. 2011. Effects of the $\mathrm{pH}$ on growth and morphology of Anabaenopsis elenkinii Miller (Cyanobacteria) isolated from the alkaline shallow lake of the Brazilian Pantanal. Fottea 11(1): 119-126.

Santos, K.R.S. \& Sant'Anna, C.L. 2010. Cianobactérias de diferentes tipos de lagoas ("salina", "salitrada" e "baía") representativas do Pantanal da Nhecolândia, MS, Brasil. Revista Brasileira de Botânica. 33(1): 61-83.

Scott, M.J. \& Jones, M.N. 2000. The biodegradation of surfactants in the environment. Biochim. Biophys. Acta. 1508: 235-251.

Silva, D.D. 2009. Revisão do gênero Planktothrix Anagnostidis \& Komarek, 1988 (Cyanobacteria/ Oscillatoriales). Tese de Doutorado. Instituto de Botânica de São Paulo - IBt. 160p.

Souza, A.G.D. 2006. Ensaios de Biodegradação de Surfactantes em Resíduos Industriais. Dissertação de Mestrado. Universidade Federal de São João Del-Rei - UFSJ. 108p.

Tonk, L.; Visser, M.; Petra, C.G.; Dittmann, E.O.F.M.; Snelder, E.; Wiedner, C.; Mur, L.R. \& Huisman, J. 2005. The microcystin composition of the Cyanobacterium Planktothrix agardii changes toward a more toxic variant with increasing light Intensity. Appl. Environ. Microb. 71(9): 5177-5181.

Tözüm-Calgan, S.R.D. \& Atay-Güneyman, N.Z. 1994. The effects of an anionic and a non-ionic surfactant on growth and nitrogen fixing ability of a cyanobacterium, Gloeocapsa. J. Environ. Sci. Health. 29(2): 355-369.

Yan, G.A.; Jiang, J.W. \& Yan, G.W. 1998. Disappearance of Linear Alkylbenzene Sulfonate from Different Cultures with Anabaena sp. HB 1017. Bull. Environ. Contam. Toxicol. 60: 329-334.

Zagatto, P.A.; Araújo, R.P.D.A.; Goldstein, E.G. \& Bertoletti, E. 1987. Avaliação do nível trófico das águas do mar pequeno. Rev. CETESB Amb. 1(2): 95-98.

Submetido: Mar3o/2014

Revisado: Julho/2014

Aceito: Setembro/2014 